\title{
A TREE ARGUMENT IN INFINITARY MODEL THEORY ${ }^{1}$
}

\author{
V. HARNIK AND M. MAKKAI
}

\begin{abstract}
A tree argument is used to show that any counterexample to Vaught's conjecture must have an uncountable model. A similar argument replaces the use of forcing by Burgess in a theorem on $\Sigma_{1}^{1}$ equivalence relations.
\end{abstract}

A formula or a sentence is one of $L_{\omega_{1} \omega}$. A sentence $\phi$ is a counterexample to Vaught's conjecture or, simply, a counterexample if it has more than $\aleph_{0}$ but less than $2^{\boldsymbol{k}_{0}}$ nonisomorphic countable models. A sentence is large if it has more than $\aleph_{0}$ nonisomorphic countable models. A large sentence is minimal if for every sentence $\psi$, either $\phi \wedge \psi$ or $\phi \wedge \neg \psi$ is not large.

THEOREM 1. Every counterexample can be strengthened to a minimal counterexample, i.e., if $\sigma$ is a counterexample, then there is a minimal counterexample $\phi$ such that $\phi \models \sigma$.

The proof of Theorem 1 uses a lemma due to Morley [8], whose formulation depends on the following (stronger than usual) notion of fragment. A set of formulas $\Delta$ is a fragment if it is closed under subformulas, substitutions of terms, finitary logical operations and if it satisfies: whenever $\phi \in \Delta, \bigvee \Theta \in \Delta$ (where $\Theta \subset \Delta)$, then $\bigvee\{\exists x \theta: \theta \in \Theta\}, \bigvee\{\phi \wedge \theta: \theta \in \Theta\}$, $\bigvee(\{\phi\} \cup \Theta)$ all belong to $\Delta$.

LEMMA 2 [8]. Let $\Delta$ be a countable fragment. If $T \subset \Delta$ is a finitely consistent set of sentences such that for all valid $\bigvee \Theta \in \Delta$ there is $a \theta \in \Theta$ which belongs to $T$, then $T$ is consistent.

Proof of Theorem 1. Assume that the theorem is false for some $\sigma$. Then it is easily seen that there is a countable fragment $\Delta$ containing $\sigma$ and such that for every large $\phi \in \Delta$ s.t. $\phi \vDash \sigma$, there is $\psi \in \Delta$ with both $\phi \wedge \psi$ and $\phi \wedge \neg \psi$ large. Let $\left\{\delta_{n}\right\}_{n<\omega}$ be an enumeration of all valid disjunctions belonging to $\Delta$. We are going to define a tree $T_{s}, s \in{ }^{<\omega} 2(=$ the set of finite sequences of 0 's and 1's) such that for all $s$ :

(a) $T_{s}$ is a finite subset of $\Delta, \sigma \in T_{s}$ and $\wedge T_{s}$ is a large sentence;

(b) $T_{s^{\wedge}\langle 0\rangle}$ and $T_{s^{\wedge}\langle 1\rangle}$ are contradictory; and

(c) if lh $s=i$ and $\delta_{i}=\bigvee \Theta$ then there are $\theta^{\prime}, \theta^{\prime \prime} \in \Theta$ s.t. $\theta^{\prime} \in T_{s^{\wedge}\langle 0\rangle}$ and $\theta^{\prime \prime} \in T_{s^{\wedge}\langle 1\rangle}$.

Received by the editors April 6, 1977.

AMS (MOS) subject classifications (1970). Primary 02B25.

'Research partially supported by the National Research Council of Canada. 
Once the tree is constructed, consider, for each $\eta \in{ }^{\omega} 2$ (= the set of infinite sequences of 0 's and 1's), the set $T_{\eta}=\cup\left\{T_{\eta \uparrow i}: i<\omega\right\}$. By (a), (c) and Lemma 2, each $T_{\eta}$ is consistent, hence has a countable model $M_{\eta}$ (which, by (a), is a model of $\sigma$ ); by (b), if $\eta \neq \eta^{\prime}$ then $M_{\eta} \cong M_{\eta^{\prime}}$. Hence $\left\{M_{\eta}: \eta \in^{\omega} 2\right\}$ is a collection of $2^{k_{0}}$ nonisomorphic countable models of $\sigma$, a contradiction to the assumption that $\sigma$ is a counterexample.

Thus, to conclude the proof, we have to indicate how to get $T_{s}$. This is done by induction on the length of $s$. Take $T_{\langle\rangle}=\{\sigma\}$. Assume that $T_{s}$ has been defined and $\operatorname{lh} s=i$. As $\wedge T_{s} \in \Delta$ is large and implies $\sigma$, there is $\psi \in \Delta$ s.t. both $\wedge T_{s} \wedge \psi$ and $\wedge T_{s} \wedge \neg \psi$ are large. If $\delta_{i}=\vee \Theta$ then any of the uncountably many models of $\wedge T_{s} \wedge \psi\left(\wedge T_{s} \wedge \neg \psi\right)$ is a model of some $\wedge T_{s} \wedge \psi \wedge \theta\left(\wedge T_{s} \wedge \neg \psi \wedge \theta\right)$, with $\theta \in \Theta$. Thus, there is $\theta^{\prime} \in \Theta\left(\theta^{\prime \prime} \in\right.$ $\Theta)$ s.t. $\wedge T_{s} \wedge \Psi \wedge \theta^{\prime}\left(\wedge T_{s} \wedge \neg \psi \wedge \theta^{\prime \prime}\right)$ is large. Take $T_{s^{\wedge}\langle 0\rangle}=T_{s} \cup$ $\left\{\psi, \theta^{\prime}\right\}$ and $T_{s^{\wedge}\langle 1\rangle}=T_{s} \cup\left\{\neg \psi, \theta^{\prime \prime}\right\}$.

The proof of Theorem 1 is now complete.

Given a counterexample $\sigma$, call a formula $\phi(\mathbf{x})$ with free variables $\mathbf{x}(\mathbf{x}$ a finite sequence) large (with respect to $\sigma$ ) if $\sigma \wedge \exists \mathbf{x} \phi(\mathbf{x})$ is large. Call $\phi(\mathbf{x})$ minimal (w.r.t. $\sigma$ ) if for all $\psi(\mathbf{x}), \phi \wedge \psi$ or $\phi \wedge \neg \psi$ is not large. Theorem 1 yields

COROllary 3. Every large (w.r.t. a given counterexample o) formula $\phi(\mathbf{x})$ can be strengthened to a minimal formula.

Proof. Notice that $\phi(x)$ is large iff $\sigma \wedge \phi(\mathbf{c})$ (a sentence in the larger language $L(c)$ with $\mathbf{c}$ a sequence of new constants) is a counterexample and that $\phi(\mathbf{x})$ is minimal w.r.t. $\sigma$ iff $\sigma \wedge \phi(\mathbf{c})$ is a minimal counterexample. Now, the assertion follows from Theorem 1 .

Now assume that $\sigma$ is a minimal counterexample. For any fragment $\Delta$ containing $\sigma$, define $T_{\Delta}=\{\phi: \phi \in \Delta$ and $\phi \wedge \sigma$ is large $\}$. If $\Delta$ is countable then, by the minimality of $\sigma, T_{\Delta}$ is consistent (all but countably many of the countable models of $\sigma$ are models of $T_{\Delta}$ ) and $\Delta$-complete. A formula $\psi(\mathbf{x}) \in$ $\Delta$ is consistent with $T_{\Delta}$ iff it is large. This observation easily yields

LEMMA 4. If $\phi(\mathbf{x})$ is minimal, then for all countable $\Delta$, if $\phi \in \Delta$ then $\phi$ is complete (in $\Delta)$ with respect to $T_{\Delta}(c f$. the definition on $p .61$ of [4]).

Call a fragment $\Delta$ closed if for every large $\phi(\mathbf{x}) \in \Delta$ there is a minimal formula $\phi^{\prime}(\mathbf{x}) \in \Delta$ s.t. $\vDash \phi^{\prime} \rightarrow \phi$. By Corollary 3 , every countable fragment can be enlarged to a closed one. If $\Delta$ is countable and closed then, by Lemma 4 and the definition of $T_{\Delta}$, there are no incompletable formulas $\psi(\mathbf{x})$ with respect to $T_{\Delta}$. Hence, $T_{\Delta}$ has a prime model (cf. [4, pp. 61-64, especially Theorem 16]). Moreover, it is easily seen that if $\Delta$ is closed then a formula $\phi \in \Delta$ is complete w.r.t. $T_{\Delta}$ iff it is minimal. Hence, each finite sequence of elements in the prime model of $T_{\Delta}$ satisfies a minimal formula belonging to $\Delta$. 
As an application, we can now prove the following result (announced in [2]).

THEOREM 5. If $\sigma$ is a counterexample then it has an uncountable model $N$. Moreover, $N$ can be so chosen as to satisfy only large sentences; thus, $N$ is not $L_{\infty \omega}$ equivalent to any countable structure.

Proof. By Theorem 1, we may assume that $\sigma$ is minimal. We define by induction an increasing chain of countable fragments $\Delta_{\alpha}$, and of countable structures $M_{\alpha}, \alpha<\omega$, s.t.:

(i) $\sigma \in \Delta_{\alpha}$, and $\Delta_{\alpha}$ is closed.

(ii) $M_{\alpha}$ is the prime model of $T_{\Delta_{\alpha}}$.

(iii) $M_{\alpha} \neq M_{\alpha+1}, M_{\alpha} \prec_{\Delta_{\alpha}} M_{\alpha+1}$ and $M_{\lambda}=\cup_{\alpha<\lambda} M_{\alpha}$ for a limit $\lambda$.

Once this is done, we shall take $N=\cup_{\alpha<\omega_{1}} M_{\alpha}$.

The inductive definition goes as follows:

Assume that $\Delta_{\alpha}$ and $M_{\alpha}$ are defined.

Let $\Delta_{\alpha+1} \supset \Delta_{\alpha}$ be a closed fragment which contains a Scott sentence $\phi$ of $M_{\alpha}$ (cf. Chapter 2 in [4]). Let $M_{\alpha+1}$ be the prime model of $T_{\Delta_{\alpha+1}}$. As $M_{\alpha+1} \vDash T_{\Delta_{\alpha}}\left(\subset T_{\Delta_{\alpha+1}}\right)$ and $M_{\alpha}$ is a prime model of $T_{\Delta_{\alpha}}, M_{\alpha}$ can be embedded in $M_{\alpha+1}$. Thus, we may take $M_{\alpha} \prec_{\Delta_{\alpha}} M_{\alpha+1}$. As the Scott sentence $\phi$ of $M_{\alpha}$ is obviously not large, $\phi \notin T_{\Delta_{\alpha+1}}$, hence $M_{\alpha+1} \vDash \neg \phi$. This shows that $M_{\alpha} \not$ $M_{\alpha+1}$, hence, $M_{\alpha} \neq M_{\alpha+1}$.

For a limit $\lambda$, take $\Delta_{\lambda}=\cup_{\alpha<\lambda} \Delta_{\alpha}$ and $M_{\lambda}=\cup_{\alpha \in \lambda} M_{\alpha}$. Then $\Delta_{\lambda}$ is obviously closed and every finite sequence of elements of $M_{\lambda}$ satisfies a minimal, hence by Lemma 4, complete (w.r.t. $T_{\Delta_{\lambda}}$ ) formula. It follows (again by Theorem 16 in [4]) that $M_{\lambda}$ is the prime model of $T_{\Delta_{\alpha}}$.

As said before, we take $N=\cup_{\alpha<\omega_{1}} M_{\alpha}$ to get an uncountable model of $\sigma$. If $N \vDash \phi$ then there is a closed and unbounded set $C \subset \omega_{1}$ s.t. $M_{\alpha} \vDash \phi$ for all $\alpha \in C$. By construction, $M_{\alpha} \simeq M_{\beta}$ whenever $\alpha \neq \beta$; hence $\left\{M_{\alpha}: \alpha \in C\right\}$ is an uncountable collection of nonisomorphic countable models of $\phi$, showing that $\phi$ is large. This completes the proof of Theorem 5.

An equivalent formulation of Theorem 5 says that, for a minimal counterexample $\sigma, T_{\Delta}$ is consistent even for uncountable fragments $\Delta$, in particular for $\Delta=L_{\omega, \omega}$.

The model $N$ constructed in the proof of Theorem 5 has the further property that each finite sequence of it satisfies a minimal formula. This implies that $N$ has Scott height $\omega_{1}$ (the Scott height of a structure is the first $\alpha$ such that for all finite sequences a of elements of $N, N \vDash \forall \mathbf{x}\left(\phi_{\mathrm{a}}^{\alpha}(\mathbf{x}) \rightarrow\right.$ $\left.\phi_{\mathrm{a}}^{\alpha+1}(\mathbf{x})\right)$ where $\phi_{\mathrm{a}}^{\alpha}$ is the $L_{\infty \omega}$ formula defined as in Chapter 2 of [4], without the restriction $\alpha<\omega_{1}$ ). Independently, Leo Harrington showed the stronger result that every counterexample has uncountable models of arbitrarily large Scott heights $\alpha<\omega_{2}$ (unpublished).

The second author of the present paper showed [5] that every counterexample has an uncountable model which is $L_{\infty}$-equivalent to a countable one. 
In a somewhat different context, we wish now to point out that the same tree argument that went into the proof of Theorem 1 can be used to replace Burgess' use in [1] of forcing in the deduction of his result on $\Sigma_{1}^{1}$ equivalence relations from Silver's theorem on $\Pi_{1}^{1}$ equivalence relation [10] (cf. also [3]).

By an equivalence relation $\mathrm{E}$ we mean one defined between countable $L$-structures which is weaker than isomorphism, i.e., if $M \cong N$ then $M(\mathrm{E}) N$. Let $L^{\prime}$ be the (two sorted) language corresponding to the naturally defined disjoint sum $M \oplus N$ of any two $L$-structures $M, N$. The equivalence relation $\mathrm{E}$ is Borel (resp. $\Sigma_{1}^{1}$ ) if for some $\sigma \in L_{\omega_{1} \omega}^{\prime}\left(\operatorname{resp} . \sigma(\mathbf{P}) \in L^{\prime}(\mathbf{P})_{\omega_{1} \omega}\right), M(\mathrm{E}) N$ iff $M \oplus N \vDash \sigma \quad(M \oplus N \vDash \exists \operatorname{Po}(\mathbf{P}))$. The following is an observation of Burgess:

Claim 6. Every $\Sigma_{1}^{1}$ equivalence relation is the intersection of $\kappa_{1}$ Borel equivalence relations.

Sketch of a model-theoretic proof. By Vaught [11] (see also [6]), there are $L_{\omega, \omega}^{\prime}$ sentences $\phi_{\alpha}, \alpha<\omega_{1}$, such that on countable structures $\exists \mathbf{P \sigma}(\mathbf{P})$ is equivalent to $\bigwedge_{\alpha<\omega_{1}} \phi_{\alpha}$. Set $M\left(\mathrm{E}_{\alpha}\right) N$ iff $M \oplus N \vDash \phi_{\alpha}$. The claim will follow if we show that $\mathrm{E}_{\alpha}$ is an equivalence relation whenever $\alpha$ is an (admissible) ordinal such that $\sigma(\mathbf{P})$ is $\mathbb{Q}$-finite for some admissible set with ord $\mathrm{d}_{\mathfrak{Q}}=\alpha$. But this is easily established using the existence of $\Sigma_{\mathcal{Q}^{-}}$saturated models, as well as the fact that, in any $\Sigma_{\mathscr{Q}^{-}}$saturated structure, $\phi_{\alpha}\left(=\wedge_{\beta<\alpha} \phi_{\beta}\right)$ is equivalent to $\exists \operatorname{Po}(\mathbf{P})$ (cf. Corollary 7.3 and the proof of 8.1 in [6]; the notion of $\Sigma_{Q^{-}}$ saturated structure comes from [9]).

Using Claim 6 and a forcing argument, Burgess deduced from Silver's aforementioned theorem that any $\Sigma_{1}^{1}$ equivalence relation has $<\kappa_{1}$ or $2^{\kappa_{0}}$ equivalence classes. This result follows from the following.

THEOREM 7. Assume that $\kappa_{0}<\kappa<2^{\kappa_{0}}$. If $\mathrm{E}$ is the intersection of $\kappa$ many Borel equivalence relations then $\mathrm{E}$ has $\leqslant \kappa$ or $2^{\kappa_{0}}$ many equivalence classes.

Proof. Let $\mathrm{E}=\bigcap_{\alpha<\kappa} \mathrm{E}_{\alpha}$, each $\mathrm{E}_{\alpha}$ a Borel equivalence relation; as is well known and easily seen, every equivalence class of any $E_{\alpha}$ is also Borel, i.e. definable by an $L_{\omega_{1} \omega}$ sentence.

Assume that $\mathrm{E}$ has $<2^{\mathrm{N}_{0}}$ equivalence classes. Then the same is true for each $E_{\alpha}$ and by (a weakened version of) Silver's theorem, $E_{\alpha}$ has $\leqslant \kappa_{0}$ equivalence classes. For every equivalence class $X$ of any $\mathrm{E}_{\alpha}, \alpha<\kappa$, select an $L_{\omega_{1} \omega}$ sentence defining it; collect all these sentences into a set $\Psi$. The properties of $\Psi$ are summed up as follows:

(i) $|\Psi| \leqslant \kappa$;

(ii) each $\psi \in \Psi$ is E-invariant, i.e., $M \models \psi$ and $M(\mathrm{E}) N$ imply that $N=\psi$; and

(iii) $\Psi$ distinguishes between the equivalence classes of E; i.e. if $\neg M(\mathrm{E}) N$, then for some $\psi \in \Psi, M \vDash \psi$ and $N \vDash \neg \psi$.

Assume next, for proof by contradiction, that $\mathrm{E}$ has $>\kappa$ equivalence classes. For $\phi \in L_{\omega, \omega}$, let $\mathcal{C}_{\phi}$ be the set of equivalence classes of $E$ having a nonempty intersection with $\operatorname{Mod}(\phi)$. Call $\sigma$ large iff $\bigodot_{\sigma}$ has power $>\kappa$. A 
simple counting argument shows:

Claim 8. For every large $\sigma$ there is $\psi \in \Psi$ s.t. both $\sigma \wedge \psi$ and $\sigma \wedge \neg \psi$ are large.

Proof. Let $\Gamma$ be the set of those sentences $\gamma$ which belong to $\Psi$ or are negations of members of $\Psi$ s.t. $\sigma \wedge \gamma$ is not large. Then

$$
e_{\sigma}=\bigcup\left\{e_{\sigma \wedge \gamma}: \gamma \in \Gamma\right\} \cup \cap\left\{e_{\sigma \wedge\urcorner_{\gamma}}: \gamma \in \Gamma\right\}
$$

If the claim is false, then the second term of the union is easily seen, by (iii) above, to contain just one element. Thus, by (i), $\left|\mathcal{C}_{\sigma}\right| \leqslant \kappa$, contradicting the largeness of $\sigma$.

Now, using Claim 8, one constructs precisely as in the proof of Theorem 1, a tree $T_{s}, s \in<\omega 2$ s.t. for all $s$ :

(a) $T_{s}$ is finite, $\sigma \in T_{s}$ and $\bigwedge T_{s}$ is large;

(b) there is $\psi \in \Psi$ s.t. $\psi \in T_{s^{\wedge}\langle 0\rangle}$ and $\neg \psi \in T_{s^{\wedge}\langle 1\rangle}$, and such that for all $\eta \in{ }^{\omega} 2, T_{\eta}=\cup_{n<\omega} T_{\eta\lceil n}$ has a model $M_{\eta}$. By (ii) and (b) above, $\neg M_{\eta}(\mathrm{E}) M_{\eta^{\prime}}$ whenever $\eta \neq \eta^{\prime}$. Thus $\mathrm{E}$ has $2^{\boldsymbol{\kappa}_{0}}$ equivalence classes, a contradiction.

We conclude by indicating a most natural example of a relation which is the intersection of $\aleph_{1}$ Borel equivalence relations. If $\mathrm{K}$ is the class of countable members of a $P C_{\omega_{1} \omega}$ class, define: $M(\mathrm{E}) N$ iff $M, N \notin \mathrm{K}$ or $M \cong N$. Again by [11], $\mathrm{K}=\bigcap_{\alpha<\omega_{1}} \mathrm{~K}_{\alpha}$ where each $\mathrm{K}_{\alpha}$ is Borel. Define: $M\left(\mathrm{E}_{\alpha}\right) N$ iff $M, N \notin \mathrm{K}_{\alpha}$ or $M \equiv_{\alpha} N$ (where $\equiv_{\alpha}$ means equivalence w.r.t. $L_{\omega_{1} \omega}$ sentences with quantifier rank $\leqslant \alpha$ ). Obviously, $\mathrm{E}=\bigcap_{\alpha<\omega_{1}} \mathrm{E}_{\alpha}$ and each $\mathrm{E}_{\alpha}$ is Borel. Thus, Theorem 7 implies Morley's result [7] that any $P C_{\omega_{1} \omega}$ class has $\leqslant \aleph_{1}$ or $2^{\kappa_{0}}$ countable nonisomorphic models. (Actually, Morley's proof contains an argument showing that the particular $\mathrm{E}_{\alpha}$ defined above satisfies Silver's theorem.)

Added MaY 30, 1977. In [12], John Burgess proves a theorem which is more general than our Theorem 7. Burgess' proof is forcing-free as well. We are indebted to the referee for this information.

\section{REFERENCES}

1. J. P. Burgess, Infinitary languages and descriptive set theory, Ph. D. Thesis, Univ. of California, Berkeley, 1974.

2. V. Harnik and M. Makkai, Some remarks on Vaught's conjecture, J. Symbolic Logic 40 (1975), 300-301 (abstract).

3. L. Harrington, A powerless proof of a theorem of Silver (manuscript).

4. H. J. Keisler, Model theory for infinitary logic, North-Holland, Amsterdam, 1971.

5. M. Makkai, An "admissible" generalization of a theorem on countable $\Sigma_{1}^{1}$ sets of reals with applications, Ann. of Math. Logic 11 (1977), 1-30.

6. __ Admissible sets and infinitary logic, Handbook of Logic (J. K. Barwise, editor), North-Holland, Amsterdam, 1977.

7. M. Morley, The number of countable models, J. Symbolic Logic 35 (1970), 14-18.

8. __ Applications of topology to $L_{\omega_{1} \omega}$, Proc. Sympos. Pure Math., vol. 25, Amer. Math. Soc., Providence, R. I., 1973, pp. 233-240.

9. J.-P. Ressayre, Models with compactness properties with respect to logics on admissible sets, Ann. of Math. Logic 11 (1977), 31-55.

10. J. Silver, Any $\Pi_{1}^{1}$ equivalence relation over $2^{\omega}$ has either $2^{\kappa_{0}}$ or $<\aleph_{0}$ equivalence classes (manuscript). 
11. R. Vaught, Descriptioe set theory in $L_{\omega, \omega}$, Lecture Notes in Math., vol. 337, Springer-Verlag; Bertin and New York, 1973, pp. 574-598.

12. J. P. Burgeses, Equioalences generated by families of Borel sets, Proc. Amer. Math. Soc. (to appear).

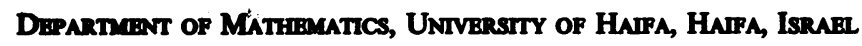

Departiant of Mathematics, MoGul Universtty, Montranl, Quibec, Canada 